Research Article

\title{
Reproducing Kernel Method for Fractional Riccati Differential Equations
}

\author{
X. Y. Li, ${ }^{1}$ B. Y. Wu, ${ }^{2}$ and R. T. Wang ${ }^{1}$ \\ ${ }^{1}$ Department of Mathematics, Changshu Institute of Technology, Suzhou, Jiangsu 215500, China \\ ${ }^{2}$ Department of Mathematics, Harbin Institute of Technology, Harbin, Heilongjiang 150001, China \\ Correspondence should be addressed to B. Y. Wu; mathwby@sina.com
}

Received 29 December 2013; Accepted 7 April 2014; Published 27 April 2014

Academic Editor: Youyu Wang

Copyright (c) $2014 \mathrm{X}$. Y. Li et al. This is an open access article distributed under the Creative Commons Attribution License, which permits unrestricted use, distribution, and reproduction in any medium, provided the original work is properly cited.

This paper is devoted to a new numerical method for fractional Riccati differential equations. The method combines the reproducing kernel method and the quasilinearization technique. Its main advantage is that it can produce good approximations in a larger interval, rather than a local vicinity of the initial position. Numerical results are compared with some existing methods to show the accuracy and effectiveness of the present method.

\section{Introduction}

This paper deals with the numerical solution of the following fractional Riccati differential equation:

$$
\begin{gathered}
u^{\alpha}(x)=p(x)+q(x) u(x)+r(x) u^{2}(x), \\
0 \leq x \leq T, 0<\alpha \leq 1, \\
u(0)=0
\end{gathered}
$$

where $u^{\alpha}(x)$ denotes the Caputo fractional derivative of order $\alpha$ and

$$
u^{\alpha}(x)=\frac{1}{\Gamma(1-\alpha)} \int_{0}^{x}(x-\tau)^{-\alpha} u^{\prime}(\tau) d \tau .
$$

Riccati differential equations arise in many fields [1]. The problem has attracted much attention and has been studied by many authors. However, deriving its analytical solution in an explicit form seems to be unlikely except for certain special situations. Recently, many numerical methods [2-9] have been proposed to solve integer order Riccati differential equations. However, the discussion on the numerical methods for fractional order Riccati differential equations is rare. Odibat and Momani [10] developed a modified homotopy perturbation method for fractional Riccati differential equations.
Li [11] presented a numerical method for fractional differential equations based on Chebyshev wavelets. Hosseinnia et al. [12] applied an enhanced homotopy perturbation method for fractional Riccati differential equations. Yüzbaşı [13] introduced a numerical method for fractional Riccati differential equations using the Bernstein polynomial. Khader [14] developed the fractional Chebyshev finite difference method for fractional Riccati differential equations. Yang and Baleanu [15], Yang et al. [16], and Baleanu et al. [17] proposed local fractional variation iteration for fractional heat conduction and wave equations.

Recently, based on the reproducing kernel theory, Cui, Geng, and Lin presented the reproducing kernel method (RKM) for linear and nonlinear operator equations [1821]. The method has been developed and applied to many problems [22-26].

The aim of this paper is to present a new method for fractional Riccati differential equations, based on the RKM and the quasilinearization technique.

The rest of the paper is organized as follows. In the next section, the quasilinearization technique is applied to fractional Riccati differential equation. The RKM for reduced linear fractional differential equations is introduced in Section 3. The numerical examples are presented in Section 4. Section 5 ends this paper with a brief conclusion. 


\section{Quasilinearization of Riccati Differential Equation (1)}

In this section, the quasilinearization technique is applied to reduce (1) to a series of linear fractional problems. Define $f(x, u)=p(x)+r(x) u^{2}$. By choosing an appropriate initial approximation $u_{0}(x)$ for the function $u(x)$ in $f(x, u)$ and expanding $f(x, u)$ around $u_{0}(x)$, it follows that

$$
f\left(x, u_{1}\right)=f\left(x, u_{0}\right)+\left.\left(u_{1}-u_{0}\right) \frac{\partial f}{\partial u}\right|_{u=u_{0}}+\cdots
$$

Generally, one can write for $k=1,2, \ldots(k=$ iteration index $)$

$$
f\left(x, u_{k}\right)=f\left(x, u_{k-1}\right)+\left.\left(u_{k}-u_{k-1}\right) \frac{\partial f}{\partial u}\right|_{u=u_{k-1}}+\cdots .
$$

Therefore, the following iteration formula for (1) can be derived:

$$
\begin{gathered}
u_{k}^{\alpha}(x)+a_{k}(x) u_{k}(x)=f_{k}(x), \quad k=1,2, \ldots, \\
u_{k}(0)=0,
\end{gathered}
$$

where $a_{k}(x)=-\left[q(x)+\left.(\partial f / \partial u)\right|_{u=u_{k-1}}\right]=-[q(x)+$ $2 r(x) u_{k-1}(x)$ and $f_{k}(x)=f\left(x, u_{k-1}\right)-\left.(\partial f / \partial u)\right|_{u=u_{k-1}}=$ $p(x)-r(x) u_{k-1}^{2}(x)$ and $u_{0}(x)$ is the initial approximation.

Clearly, to solve (1), it suffices for us to solve the series of linear problem (5).

\section{Method for Solving Linear Fractional Problem (5)}

To illustrate how to solve (5) we consider the problem of solving

$$
\begin{gathered}
L v(x)=v^{\alpha}(x)+a(x) v(x)=f(x), \quad 0<x<T, \\
v(0)=0,
\end{gathered}
$$

where $a(x)$ and $f(x)$ are continuous.

By the definition of Caputo fractional derivative, (6) is equivalent to the following equation:

$$
\begin{aligned}
\frac{1}{\Gamma(1-\alpha)} \int_{0}^{t}(t-\tau)^{-\alpha} v^{\prime}(\tau) d \tau+a(t) v(t) & =f(t), \\
0 & \leq t \leq T,
\end{aligned}
$$

$$
v(0)=0 .
$$

If $u(t) \in C^{1}[0, T]$, then the improper integral $\int_{0}^{t}(t-$ $\tau)^{-\alpha} v^{\prime}(\tau) d \tau$ exists and

$$
\begin{aligned}
\int_{0}^{t}(t & -\tau)^{-\alpha} v^{\prime}(\tau) d \tau \\
& =\int_{0}^{t} \frac{v^{\prime}(\tau)-v^{\prime}(t)}{(t-\tau)^{\alpha}} d \tau+v^{\prime}(t) \int_{0}^{t}(t-\tau)^{-\alpha} d \tau \\
& =\frac{t^{1-\alpha} v^{\prime}(t)}{1-\alpha}-\int_{0}^{t} \frac{v^{\prime}(t)-v^{\prime}(\tau)}{(t-\tau)^{\alpha}} d \tau \\
& =\frac{t^{1-\alpha} v^{\prime}(t)}{1-\alpha}-\int_{0}^{t} g(\tau, v) d \tau
\end{aligned}
$$

TABLE 1: Comparison of the numerical solutions with the other methods for $\alpha=0.75$.

\begin{tabular}{lccc}
\hline$x$ & Ours & {$[10]$} & {$[11]$} \\
\hline 0.20 & 0.469516 & 0.428892 & 0.584307 \\
0.40 & 0.933596 & 0.891404 & 1.024974 \\
0.50 & 1.14488 & 1.132763 & 1.198621 \\
0.60 & 1.33098 & 1.370240 & 1.349150 \\
0.80 & 1.62153 & 1.794879 & 1.599235 \\
1.00 & 1.81865 & 2.087384 & 1.801763 \\
\hline
\end{tabular}

Define

$$
g(\tau, v)= \begin{cases}\frac{v^{\prime}(t)-v^{\prime}(\tau)}{(t-\tau)^{\alpha}}, & \tau \neq t \\ 0, & \tau=t\end{cases}
$$

This gives us a continuous function on $[0, t]$ and then integral $\int_{0}^{t} g(\tau, v) d \tau$ also exists and $\int_{0}^{t}\left(\left(v^{\prime}(t)-v^{\prime}(\tau)\right) /(t-\tau)^{\alpha}\right) d \tau=$ $\int_{0}^{t} g(\tau, v) d \tau$.

Therefore, (6) can be converted into

$$
\frac{1}{\Gamma(1-\alpha)}\left(\frac{t^{1-\alpha} v^{\prime}(t)}{1-\alpha}-\int_{0}^{t} g(\tau, v) d \tau\right)+a(t) v(t)=f(t)
$$

$$
0 \leq t \leq T
$$

$$
v(0)=0 \text {. }
$$

Applying Hermite's quadrature formula to $\int_{0}^{t}\left(\left(v^{\prime}(t)-\right.\right.$ $\left.\left.v^{\prime}(\tau)\right) /(t-\tau)^{\alpha}\right) d \tau$, one obtains

$$
\int_{0}^{t} g(\tau, v) d \tau=\frac{\pi t}{2 M} \sum_{k=1}^{M} \bar{g}\left(x_{k}, u\left(x_{k}\right)\right) \sqrt{1-x_{k}^{2}}
$$

where $\bar{g}(x, u(x))=g((t / 2)(1+x), u((t / 2)(1+x))), M$ is the number of nodes, and $x_{k}=\cos ((2 k-1) / 2 M), k=1, \ldots, M$.

Then (6) can be further equivalently approximated to

$$
\begin{aligned}
& \frac{1}{\Gamma(1-\alpha)} \\
& \times\left(\frac{t^{1-\alpha} v^{\prime}(t)}{1-\alpha}-\frac{\pi t}{2 M} \sum_{k=1}^{M} \bar{g}\left(x_{k}, v\left(x_{k}\right)\right) \sqrt{1-x_{k}^{2}}\right) \\
& +a(t) v(t)=f(t), \quad 0 \leq t \leq T, \\
& v(0)=0 .
\end{aligned}
$$

To apply the RKM to (12), it is necessary to construct the following reproducing kernel Hilbert space $W^{3}[0, T]$.

Definition 1. $W^{3}[0, T]=\left\{u(x) \quad \mid u^{\prime \prime}(x)\right.$ is an absolutely continuous real value function, $u^{(3)}(x) \in L^{2}[0, T]$, 


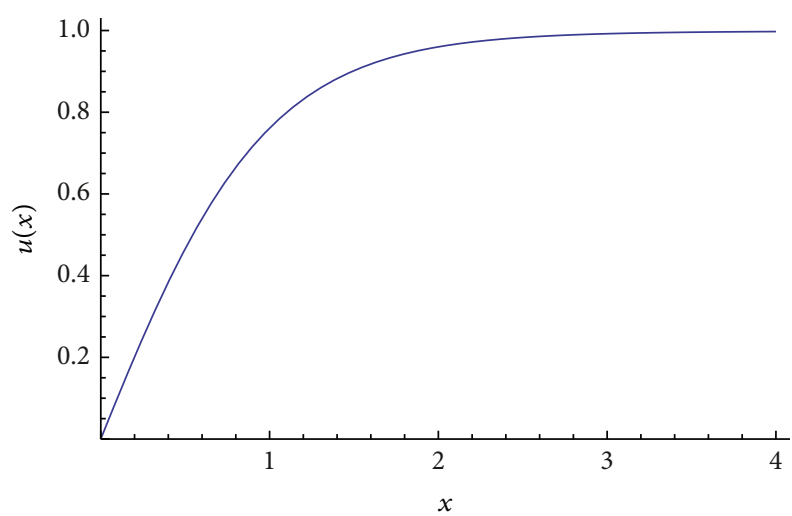

(a)

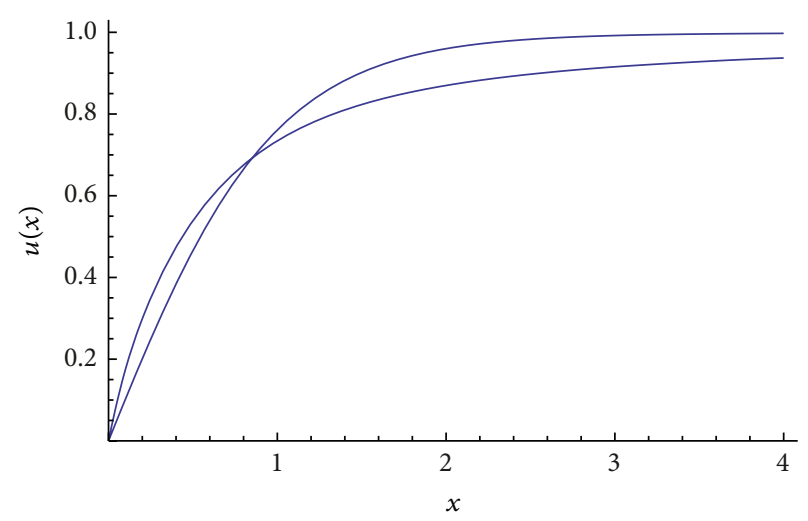

(b)

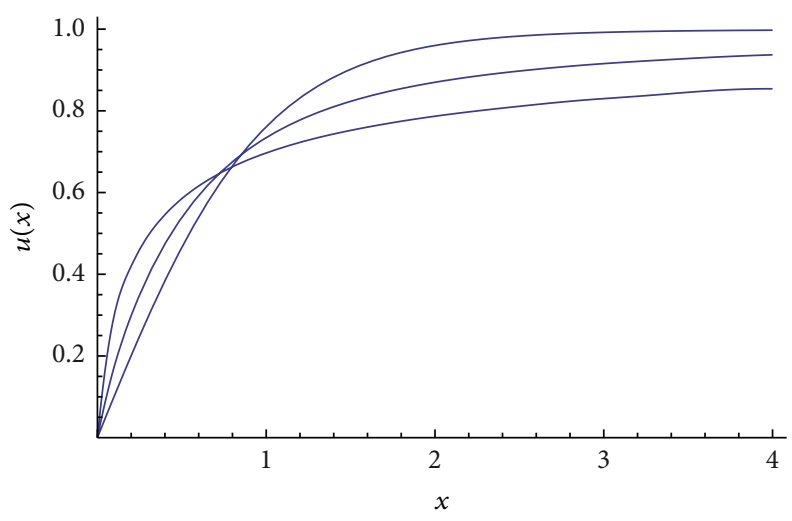

(c)

FIGURE 1: The behavior of approximate solution with different values of $\alpha$ ((a) $\alpha=0.99$; (b) $\alpha=0.75,0.99$; (c) $\alpha=0.5,0.75,0.99$ ).

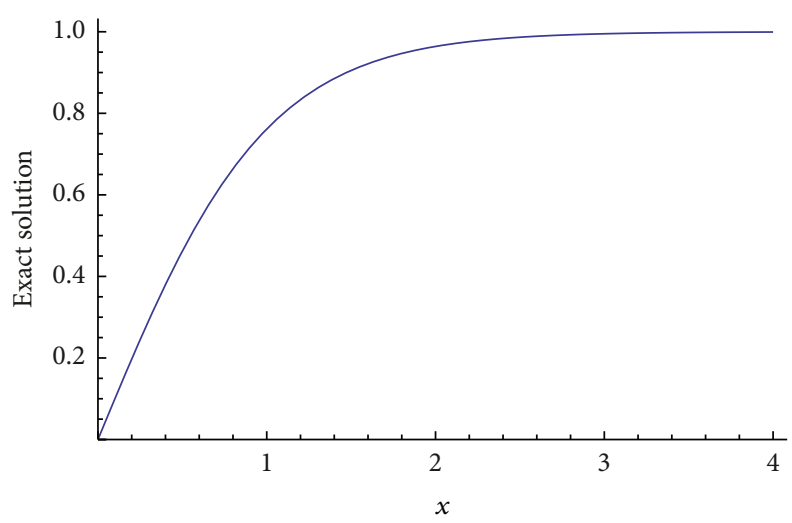

(a)

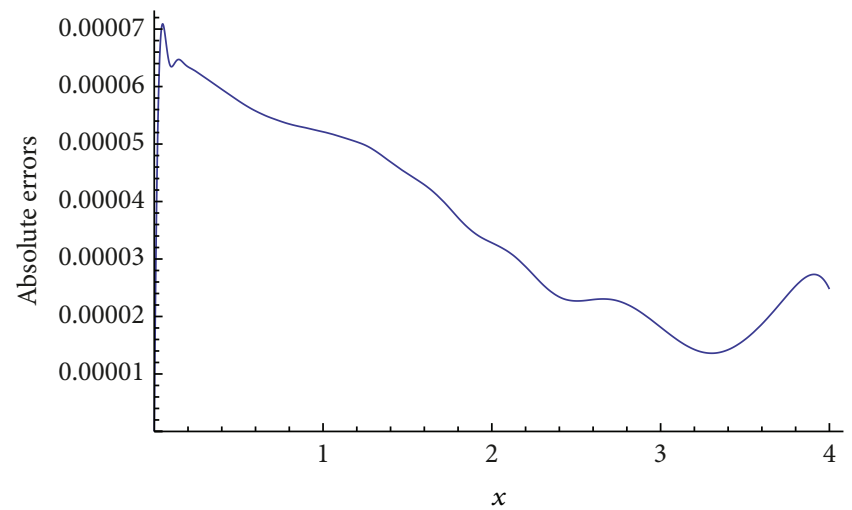

(b)

FIgURE 2: Comparison of approximate solutions with the exact solutions for $\alpha=1$ ((a) exact solution; (b) absolute errors).

$u(0)=0\}$. The inner product and norm in $W^{3}[0, T]$ are given, respectively, by

$$
\begin{aligned}
& (u(y), v(y))_{3} \\
& =u(0) v(0)+u^{\prime}(0) v^{\prime}(0)+u^{\prime \prime}(0) v^{\prime \prime}(0)+\int_{0}^{T} u^{(3)} v^{(3)} d y, \\
& \|u\|_{3}=\sqrt{(u, u)_{3}}, \quad u, v \in W^{3}[0, T] .
\end{aligned}
$$

Theorem 2. $W^{3}[0, T]$ is a reproducing kernel space and its reproducing kernel is

$$
k(x, y)= \begin{cases}k_{1}(x, y), & y \leq x \\ k_{1}(y, x), & y>x\end{cases}
$$

where $k_{1}(x, y)=y^{2}\left(7 x y^{4}-y^{5}+35 x^{3} y(4+y)-\right.$ $\left.21 x^{2}\left(-60+y^{3}\right)\right) / 5040$. 
TABLE 2: Comparison of the numerical solutions with the other methods for $\alpha=0.90$.

\begin{tabular}{lcccc}
\hline$x$ & Ours & {$[13]$} & IABMM [12] & MHPM [10] \\
\hline 0.20 & 0.312985 & 0.314869 & - & - \\
0.40 & 0.695357 & 0.697544 & - & - \\
0.50 & 0.901484 & 0.903695 & 0.8621 & 0.9010 \\
0.60 & 1.10576 & 1.107866 & - & - \\
0.80 & 1.47606 & 1.477707 & - & - \\
1.00 & 1.76417 & 1.764520 & 1.7356 & 1.8720 \\
\hline
\end{tabular}

TABLE 3: Numerical results for $\alpha=0.99,1$ on $[0,4]$.

\begin{tabular}{lccc}
\hline$x$ & Ours $(\alpha=0.99)$ & Ours $(\alpha=1)$ & Exact solution $(\alpha=1)$ \\
\hline 0.5 & 0.769552 & 0.756446 & 0.756014 \\
1.0 & 1.69828 & 1.68978 & 1.689500 \\
1.5 & 2.19159 & 2.19599 & 2.195630 \\
2.0 & 2.35063 & 2.35838 & 2.357770 \\
2.5 & 2.39373 & 2.40046 & 2.40028 \\
3.0 & 2.40539 & 2.41058 & 2.41081 \\
3.5 & 2.40899 & 2.41302 & 2.41339 \\
4.0 & 2.41047 & 2.41374 & 2.41401 \\
\hline
\end{tabular}

TABLE 4: Comparison of the numerical solutions with the other methods for $\alpha=0.75$.

\begin{tabular}{lcccc}
\hline$x$ & Ours & {$[13]$} & IABMM [12] & MHPM [10] \\
\hline 0.20 & 0.307359 & 0.3099755 & 0.3117 & 0.3138 \\
0.40 & 0.480346 & 0.4816318 & 0.4855 & 0.4929 \\
0.60 & 0.597542 & 0.5977827 & 0.6045 & 0.5974 \\
0.80 & 0.679657 & 0.6788495 & 0.6880 & 0.6604 \\
1.00 & 0.738213 & 0.7368368 & 0.7478 & 0.7183 \\
\hline
\end{tabular}

TABLE 5: Comparison of the numerical solutions with the other methods for $\alpha=0.90$.

\begin{tabular}{lcccc}
\hline$x$ & Ours & {$[13]$} & IABMM [12] & MHPM [10] \\
\hline 0.20 & 0.237652 & 0.2387891 & 0.2393 & 0.2391 \\
0.40 & 0.421766 & 0.4225830 & 0.4234 & 0.4229 \\
0.60 & 0.565673 & 0.5661715 & 0.5679 & 0.5653 \\
0.80 & 0.674464 & 0.6746270 & 0.6774 & 0.6740 \\
1.00 & 0.754632 & 0.7545890 & 0.7584 & 0.7569 \\
\hline
\end{tabular}

Definition 3. $W^{1}[0, T]=\{u(x) \mid u(x)$ is an absolutely continuous real value function, $\left.u^{\prime}(x) \in L^{2}[0, T]\right\}$. The inner product and norm in $W^{1}[0, T]$ are given, respectively, by

$$
\begin{gathered}
(u(y), v(y))_{1}=u(0) v(0)+\int_{0}^{T} u^{\prime} v^{\prime} d y, \\
\|u\|_{1}=\sqrt{(u, u)_{1}}, \quad u, v \in W^{1}[0, T] .
\end{gathered}
$$

TABLE 6: Comparison of the numerical solutions with the other methods for $\alpha=1.0$.

\begin{tabular}{lcccc}
\hline$x$ & Exact & Ours & {$[13]$} & MHPM [10] \\
\hline 0.20 & 0.197375 & 0.19738 & 0.197375 & 0.197375 \\
0.40 & 0.379949 & 0.379956 & 0.379948 & 0.379944 \\
0.60 & 0.53705 & 0.537061 & 0.537049 & 0.536857 \\
0.80 & 0.664037 & 0.664053 & 0.664036 & 0.661706 \\
1.00 & 0.761594 & 0.761618 & 0.761594 & 0.746032 \\
\hline
\end{tabular}

Theorem 4. $W^{1}[0, T]$ is a reproducing kernel space and its reproducing kernel is

$$
\bar{k}(x, y)= \begin{cases}1+y, & y \leq x, \\ 1+x, & y>x .\end{cases}
$$

Put

$$
\begin{aligned}
& \operatorname{Lv}(x) \\
& \begin{aligned}
\frac{1}{\Gamma(1-\alpha)}( & \frac{t^{1-\alpha} v^{\prime}(t)}{1-\alpha}-\frac{\pi t}{2 M} \\
& \left.\times \sum_{k=1}^{M} \bar{g}\left(x_{k}, v\left(x_{k}\right)\right) \sqrt{1-x_{k}^{2}}\right)+a(t) v(t) .
\end{aligned}
\end{aligned}
$$

Clearly, $L: W^{3}[0, T] \rightarrow W^{1}[0, T]$ is a bounded linear operator. Put $\varphi_{i}(x)=\bar{k}\left(x, x_{i}\right)$ and $\psi_{i}(x)=L^{*} \varphi_{i}(x)$, where $L^{*}$ is the adjoint operator of $L$. The orthonormal system $\left\{\bar{\psi}_{i}(x)\right\}_{i=1}^{\infty}$ of $W^{3}[0, T]$ can be derived from Gram-Schmidt orthogonalization process of $\left\{\psi_{i}(x)\right\}_{i=1}^{\infty}$,

$$
\bar{\psi}_{i}(x)=\sum_{k=1}^{i} \beta_{i k} \psi_{k}(x), \quad\left(\beta_{i i}>0, i=1,2, \ldots\right) .
$$

Theorem 5. If $\left\{x_{i}\right\}_{i=1}^{\infty}$ is dense on $[0, T]$, then $\left\{\psi_{i}(x)\right\}_{i=1}^{\infty}$ is the complete system of $W^{3}[0, T]$.

Theorem 6. If $\left\{x_{i}\right\}_{i=1}^{\infty}$ is dense on $[0, T]$ and the solution of (12) is unique, then the solution of (12) is

$$
v(t)=\sum_{i=1}^{\infty} \sum_{k=1}^{i} \beta_{i k} f\left(x_{k}\right) \bar{\psi}_{i}(t) .
$$

Now, an approximate solution $V_{N}(x)$ of (6) can be obtained by the $N$-term intercept of the exact solution $v(x)$ and

$$
v_{N}(x)=\sum_{i=1}^{N} \sum_{k=1}^{i} \beta_{i k} f\left(x_{k}\right) \bar{\psi}_{i}(t) .
$$

Similarly, the approximate solutions $u_{k}(x)$ can be obtained:

$$
u_{k, N}(x)=\sum_{j=1}^{N} A_{j} \bar{\psi}_{j}(x),
$$

where $A_{j}=\sum_{l=1}^{j} \beta_{j l} f_{k}\left(x_{l}\right)$. 


\section{Numerical Examples}

Example 1. Consider the following fractional Riccati differential equation [10-13]:

$$
\begin{gathered}
u^{\alpha}(x)=1+2 u(x)-u^{2}(x), \\
0 \leq x \leq T, 0<\alpha \leq 1, \\
u(0)=0 .
\end{gathered}
$$

The exact solution for $\alpha=1$ can be easily determined to be

$$
u(x)=1+\sqrt{2} \tanh \left(\sqrt{2} x+\frac{\log ((-1+\sqrt{2}) /(1+\sqrt{2}))}{2}\right) .
$$

Applying the proposed method, taking $T=1, k=3, M=$ $30, N=50$, the numerical results compared with other methods are listed in Tables 1 and 2. Taking $T=4, k=$ 3, $M=30, N=50$, the numerical results on $[0,4]$ are listed in Table 3. From Table 3, it is easily found that the present approximations are effective for a larger interval, rather than a local vicinity of the initial position.

Example 2. Consider the following fractional Riccati differential equation [10-14]:

$$
\begin{gathered}
u^{\alpha}(x)=1+u^{2}(x), \quad 0 \leq x \leq T, 0<\alpha \leq 1 \\
u(0)=0 .
\end{gathered}
$$

The exact solution for $\alpha=1$ can be easily determined to be

$$
u(x)=\frac{e^{2 x}-1}{e^{2 x}+1} .
$$

According to the present method, taking $T=1, k=$ $3, M=50, N=50$, the numerical results compared with other methods are given in Tables 4,5 , and 6 . Taking $T=4, k=5, M=50, N=80$, the numerical results on $[0,4]$ are shown in Figures 1 and 2. From these figures we can conclude that the obtained numerical solutions are in excellent agreement with the exact solution for a larger interval.

\section{Conclusion}

In this paper, combining the RKM, the numerical integral, and quasilinearization techniques, a new numerical method is proposed for fractional Riccati differential equations. The main advantage of this method is that it can provide accurate numerical approximations on a larger interval. Numerical results compared with the existing methods show that the present method is a powerful method for solving fractional Riccati differential equations.

\section{Conflict of Interests}

The authors declare that there is no conflict of interests regarding the publication of this paper.

\section{Acknowledgment}

This work was supported by the National Natural Science Foundation of China (Grant nos. 11326237, 11271100, and 11126222).

\section{References}

[1] W. T. Reid, Riccati Differential Equations, Academic Press, New York, NY, USA, 1972.

[2] M. A. El-Tawil, A. A. Bahnasawi, and A. Abdel-Naby, "Solving Riccati differential equation using Adomian's decomposition method," Applied Mathematics and Computation, vol. 157, no. 2, pp. 503-514, 2004.

[3] M. Lakestani and M. Dehghan, "Numerical solution of Riccati equation using the cubic B-spline scaling functions and Chebyshev cardinal functions," Computer Physics Communications, vol. 181, no. 5, pp. 957-966, 2010.

[4] F. Z. Geng, Y. Z. Lin, and M. G. Cui, "A piecewise variational iteration method for Riccati differential equations," Computers \& Mathematics with Applications, vol. 58, no. 11-12, pp. 25182522, 2009.

[5] B.-Q. Tang and X.-F. Li, "A new method for determining the solution of Riccati differential equations," Applied Mathematics and Computation, vol. 194, no. 2, pp. 431-440, 2007.

[6] A. Ghorbani and S. Momani, "An effective variational iteration algorithm for solving Riccati differential equations," Applied Mathematics Letters, vol. 23, no. 8, pp. 922-927, 2010.

[7] S. Momani and N. Shawagfeh, "Decomposition method for solving fractional Riccati differential equations," Applied Mathematics and Computation, vol. 182, no. 2, pp. 1083-1092, 2006.

[8] S. Abbasbandy, "A new application of He's variational iteration method for quadratic Riccati differential equation by using Adomian's polynomials," Journal of Computational and Applied Mathematics, vol. 207, no. 1, pp. 59-63, 2007.

[9] F. Mohammadi and M. M. Hosseini, "A comparative study of numerical methods for solving quadratic Riccati differential equations," Journal of the Franklin Institute. Engineering and Applied Mathematics, vol. 348, no. 2, pp. 156-164, 2011.

[10] Z. Odibat and S. Momani, "Modified homotopy perturbation method: application to quadratic Riccati differential equation of fractional order," Chaos, Solitons \& Fractals, vol. 36, no. 1, pp. 167-174, 2008.

[11] Y. L. Li, "Solving a nonlinear fractional differential equation using Chebyshev wavelets," Communications in Nonlinear Science and Numerical Simulation, vol. 15, no. 9, pp. 2284-2292, 2010.

[12] S. H. Hosseinnia, A. Ranjbar, and S. Momani, "Using an enhanced homotopy perturbation method in fractional differential equations via deforming the linear part," Computers of Mathematics with Applications, vol. 56, no. 12, pp. 3138-3149, 2008.

[13] S. Yüzbaş1, "Numerical solutions of fractional Riccati type differential equations by means of the Bernstein polynomials," Applied Mathematics and Computation, vol. 219, no. 11, pp. 6328-6343, 2013.

[14] M. M. Khader, "Numerical treatment for solving fractional Riccati differential equation," Journal of the Egyptian Mathematical Society, vol. 21, no. 1, pp. 32-37, 2013.

[15] X. J. Yang and D. Baleanu, "Fractal heat conduction problem solved by local fractional variation iteration method," Thermal Science, vol. 17, no. 2, pp. 625-628, 2013. 
[16] X.-J. Yang, D. Baleanu, and W.-P. Zhong, "Approximate solutions for diffusion equations on Cantor space-time," Proceedings of the Romanian Academy, A: Mathematics, Physics, Technical Sciences, Information Science, vol. 14, no. 2, pp. 127-133, 2013.

[17] D. Baleanu, J. A. Tenreiro Machado, C. Cattani, M. C. Baleanu, and X.-J. Yang, "Local fractional variational iteration and decomposition methods for wave equation on Cantor sets within local fractional operators," Abstract and Applied Analysis, vol. 2014, Article ID 535048, 6 pages, 2014.

[18] M. G. Cui and Y. Z. Lin, Nonlinear Numerical Analysis in Reproducing Kernel Space, Nova Science Publishers, 2009.

[19] F. Z. Geng and M. G. Cui, "Solving a nonlinear system of second order boundary value problems," Journal of Mathematical Analysis and Applications, vol. 327, no. 2, pp. 1167-1181, 2007.

[20] F. Z. Geng, "A numerical algorithm for nonlinear multipoint boundary value problems," Journal of Computational and Applied Mathematics, vol. 236, no. 7, pp. 1789-1794, 2012.

[21] F. Z. Geng and S. P. Qian, "Reproducing Kernel method for singularly perturbed turning point problems having twin boundary layers," Applied Mathematics Letters, vol. 26, no. 10, pp. 998-1004, 2013.

[22] M. Inc, A. Akgül, and A. Kiliçman, "A novel method for solving $\mathrm{KdV}$ equation based on reproducing Kernel Hilbert space method," Abstract and Applied Analysis, vol. 2013, Article ID 578942, 11 pages, 2013.

[23] M. Mohammadi and R. Mokhtari, "Solving the generalized regularized long wave equation on the basis of a reproducing Kernel space," Journal of Computational and Applied Mathematics, vol. 235, no. 14, pp. 4003-4014, 2011.

[24] G. Akram and H. U. Rehman, "Numerical solution of eighth order boundary value problems in reproducing Kernel space," Numerical Algorithms, vol. 62, no. 3, pp. 527-540, 2013.

[25] O. Abu Arqub, M. Al-Smadi, and S. Momani, "Application of reproducing Kernel method for solving nonlinear FredholmVolterra integrodifferential equations," Abstract and Applied Analysis, vol. 2012, Article ID 839836, 16 pages, 2012.

[26] W. Y. Wang, B. Han, and M. Yamamoto, "Inverse heat problem of determining time-dependent source parameter in reproducing Kernel space," Nonlinear Analysis. Real World Applications, vol. 14, no. 1, pp. 875-887, 2013. 


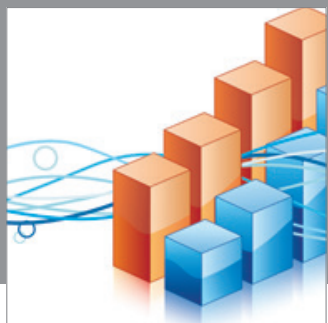

Advances in

Operations Research

mansans

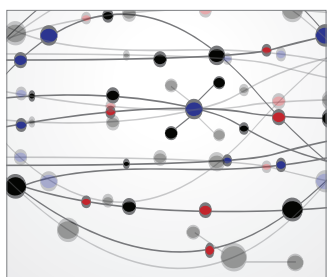

The Scientific World Journal
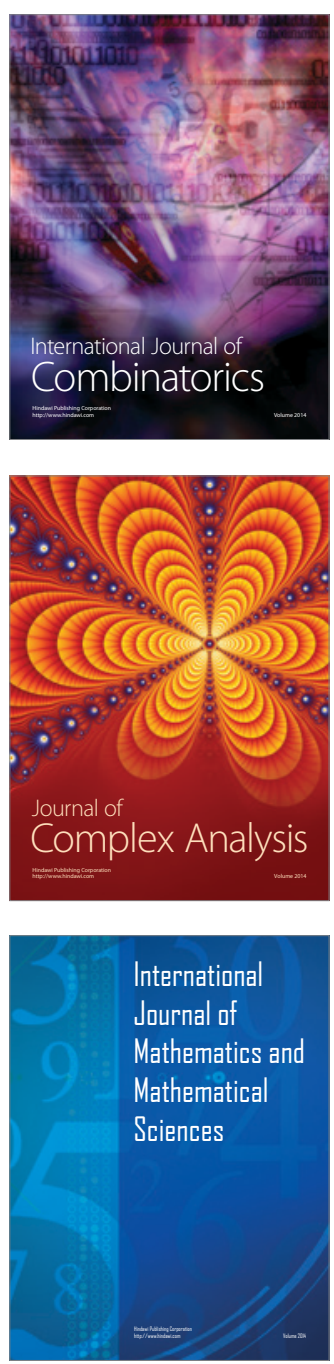
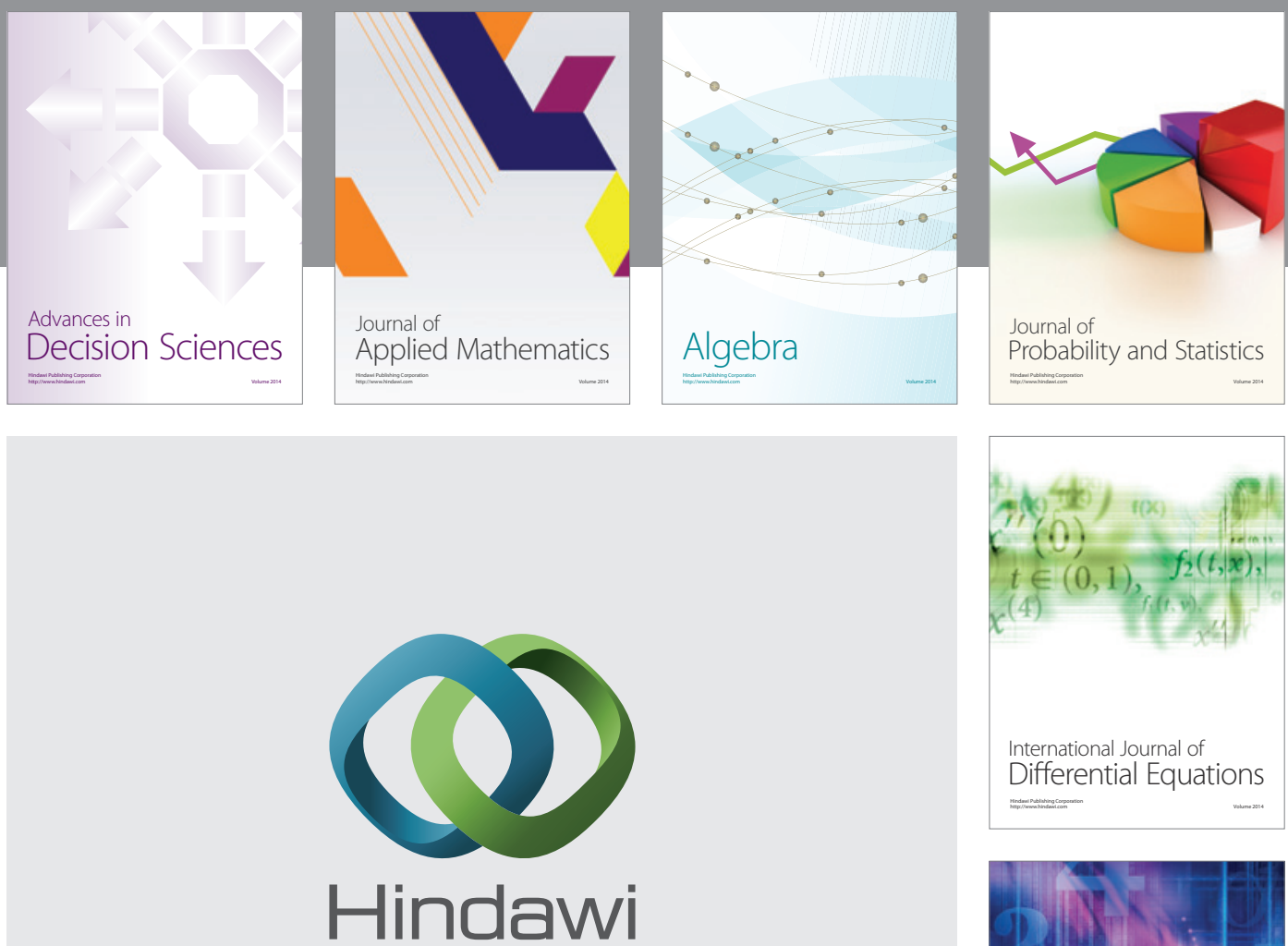

Submit your manuscripts at http://www.hindawi.com
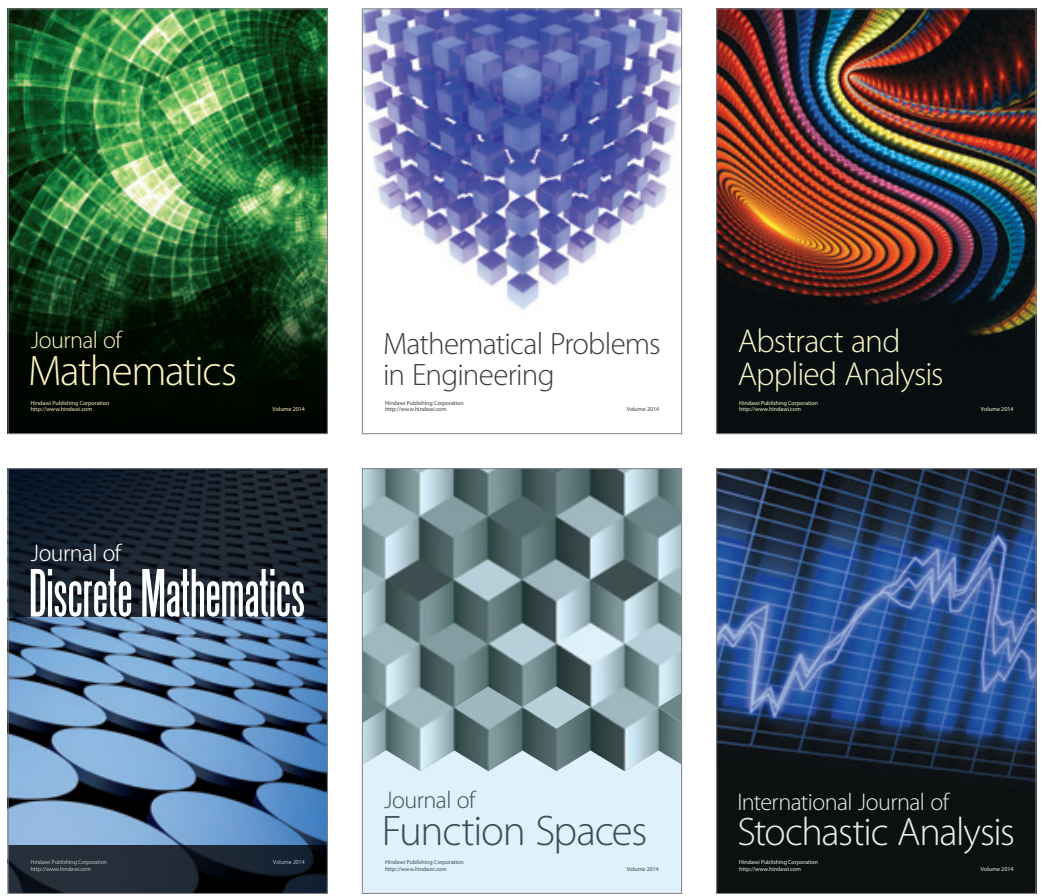

Journal of

Function Spaces

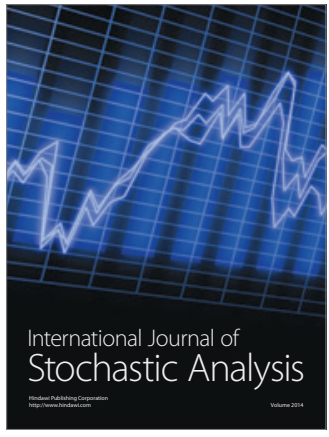

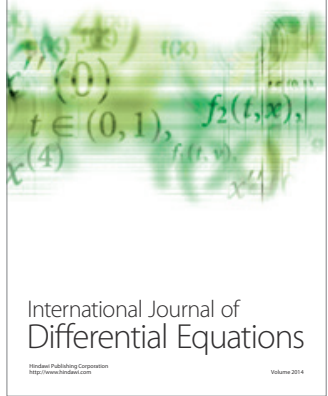
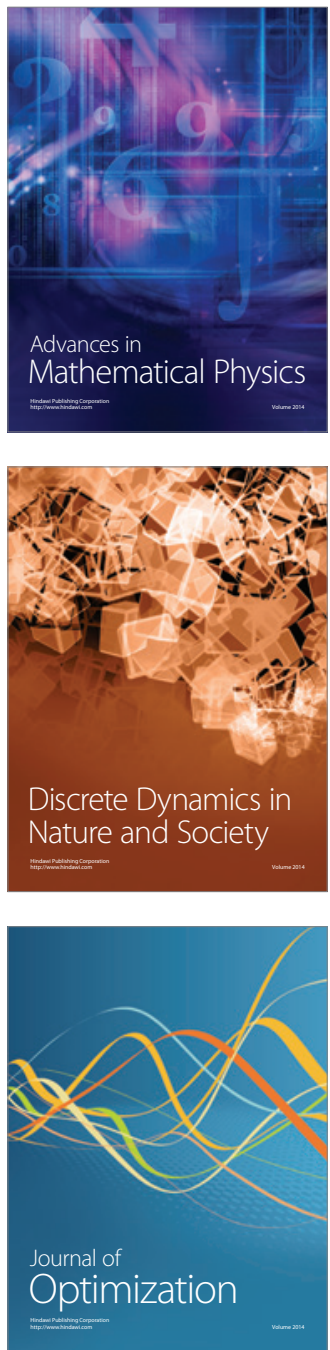